Conclusions Blood eosinophils in patients with SSc display a diverse phenotype depending on disease duration. In early disease, surface marker expression on eosinophils is associated with disease activity and severity.

\section{A2.13 PHENOTYPIC AND MOLECULAR PROFILE OF INNATE LYMPHOID CELLS IN CHRONIC SYNOVIAL INFLAMMATION}

doi:10.1136/annrheumdis-2013-203215.13

1,2Hulda Sigridur Hreggvidsdottir, ${ }^{2} J e n n y$ Mjösberg, ${ }^{2} J o c h e m$ Bernink, 'Dominique Baeten, ${ }^{2}$ Hergen Spits. 'Department of Clinical Immunology and Rheumatology; ${ }^{2}$ Tytgat Institute for Liver and Intestinal Research, Academic Medical Center/University of Amsterdam, Amsterdam, The Netherlands

Background and Objective Innate lymphoid cells (ILCs) represent a novel family of effector and regulatory cells in innate immunity and tissue remodelling. The family comprises several phenotypically and functionally distinct subsets that produce various cytokines such as IL-22, IL-17, IFN $\gamma$, TNF, IL-13 and IL-5, of which many have been shown to be important in arthritis pathogenesis.

The IL-17 and IL-22 producing ILCs are of major interest as they are implicated in chronic gut inflammation. Based on the broad clinical overlap between inflammatory bowel disease and spondyloarthritis (SpA) and the clinical importance of IL-17 in SpA we hypothesise that IL-17 and IL-22 producing ILCs contribute to inflammation and remodelling in SpA synovitis. As these cells have never been described in the joint our first aim was to characterise ILC in chronic inflammatory arthritis.

Material and Methods ILCs (lineage negative, $\mathrm{CD} 45^{+} \mathrm{CD} 127^{+}$) were analysed and sorted by flow cytometry from synovial tissue and fluid from rheumatoid arthritis (RA) and SpA patients as well as in blood from SpA patients and healthy donors. mRNA expression of sorted and expanded cells was analysed by qPCR.

Results ILCs were identified in blood as well as in synovial tissue and fluid from both RA and SpA patients. The frequency of ILCs was higher in the inflamed joint (0.5-3.3\% of the lymphocyte population) than in the peripheral blood compartment $(0.1 \%)$. In blood, there was no marked difference in the frequency of the different ILC subset between healthy controls $(n=10)$ and SpA patients $(n=5)$. In the inflamed joint, the ILC3 (CRTH2-NKp44+ckit+) and ILC1 (CRTH2-NKp44-ckit-) populations, previously shown to express IL-22 and IFN $\gamma$ respectively in other tissues, were present in all samples whereas the Th2 cytokine expressing ILC2s (CRTH2+) were found in very low frequencies. Frequencies of ILC subpopulations varied considerably between patients and no differences could be detected between RA and SpA patients. qPCR analysis of expanded cells revealed that ILC1 expressed TBX21 whereas ILC3 expressed RORC. Accordingly, stimulated ILC3 expressed transcripts for both IL-23R and IL-22 but not IL-17.

Conclusions ILC1 and ILC 3 are present in the chronically inflamed joint and express the key transcription factors associated with specific cytokine profiles. These data indicate that ILC could contribute to local cytokine-driven immune alterations in SpA and RA.

\section{A2.14 POTENTIAL IN VITRO IMMUNOMODULATORY EFFECTS OF THE RECOMBINANT HUMAN ALPHA-ENOLASE ON PERIPHERAL BLOOD MONONUCLEAR CELLS (PBMCS) FROM HEALTHY DONORS}

doi:10.1136/annrheumdis-2013-203215.14

1.* C Guillou, ${ }^{2} \mathrm{G}$ Avenel, ' $\mathrm{C}$ Derambure, ${ }^{2} \mathrm{M}$ Verdet, ${ }^{1} \mathrm{ML}$ Golinski, ${ }^{1} \mathrm{M}$ Hiron, ${ }^{1} \mathrm{~S}$ Adriouch, ${ }^{1} 0$ Boyer, ${ }^{2} \mathrm{~T}$ Lequerré, ${ }^{2} \mathrm{O}$ Vittecoq. ${ }^{1}$ INSERM U905, Institute for Biomedical Research, University of Rouen, Rouen, France; ${ }^{2}$ Department of Rheumatology, Rouen University Hospital and INSERM U905, Institute for Biomedical Research, University of Rouen, Rouen, France

*Mail: clement.guillou@etu.univ-rouen.fr
Background Identification of autoantibodies associated with rheumatoid arthritis has been of major interest. In this context, we have previously identified for the first time $\alpha$-enolase (ENO) as a new auto-antigen in early RA. ENO is an evolutionary conserved protein involved both in glycolysis pathway and as a plasminogen receptor which confer it a role in anti-infectious inflammatory response. In vivo, preliminary studies showed that ENO had immunomodulatory effect in the collagen induced arthritis mouse model. [1] To better understand the immunological mechanisms of ENO, the aim of this in vitro study was to determine the effects of ENO on PBMCs from healthy donors.

Methods In one hand, PBMCs or different cell types (monocytes, $B$ and $T$ cells, and immature dendritic cells [iDC]) $(n=3)$ were cultured with ENO $(20 \mu \mathrm{g} / \mathrm{mL})$ or Bovine Serum Albumin $(20 \mu \mathrm{g} / \mathrm{mL})$. TNF $\alpha$ and IL-10 production was measured in the supernatants by ELISA at different times. On the other hand, TNF $\alpha$ and IL-10 production were evaluated in PBMCs, monocytes or B and T cells after LPS stimulation and pre-incubation with ENO for $24 \mathrm{~h}(\mathrm{n}=3)$.

Cytometric analyses have evaluated the ability of ENO to inhibit the differentiation of monocytes into iDC. Before differentiation into iDC (GM-CSF and IL-4), monocytes $\left(1.10^{6}\right.$ cells $\left./ \mathrm{mL}\right)$ were incubated with ENO $(20$ or $50 \mu \mathrm{g} / \mathrm{mL}$ ) for $24 \mathrm{~h}$.

Results In cultures of PBMCs, monocytes or iDC, ENO induces, dose dependently, an early production of TNF $\alpha$ followed by extended secretion of IL-10. PBMCs or individual cells (monocytes, B and T cells) stimulated by LPS secreted successively TNF $\alpha$ and IL-10, while PBMCs or individual cells, stimulated by LPS but previously incubated with ENO for $24 \mathrm{~h}$ did not secrete these cytokines.

In contrast to LPS, ENO did not induce differentiation of immature dendritic cells into mature cells. But ENO has not the capacity to inhibit differentiation from monocytes to iDC.

Conclusions This study suggests that ENO has no pro-inflammatory effect unlike LPS. Indeed, ENO might have immunomodulatory properties via IL-10 production. Others studies focused on an extended cytokine panel and different signalling pathways are underway to better understand the immunological mechanisms induced by ENO.

Reference

1. C Guillou et al, Arthritis and Rheumatism 2011; 63:S815

\section{A2.15 RELATIVE OVEREXPRESSION OF TRANSMEMBRANE VERSUS SOLUBLE TNF IN HUMAN AND EXPERIMENTAL SPONDYLOARTHRITIS}

doi:10.1136/annrheumdis-2013-203215.15

Leonie M van Duivenvoorde, Carmen A Ambarus, Huriatul Masdar, Melissa N van Tok, Paul P Tak, Nataliya G Yeremenko, Dominique L Baeten. Department of Clinical Immunology and Rheumatology, Department of Experimental Immunology, Academic Medical Center, University of Amsterdam, Amsterdam, The Netherlands

Background Macrophages and their pro-inflammatory cytokines, including TNF, are pivotal mediators of chronic synovitis in rheumatoid arthritis (RA) as well as spondyloarthritis (SpA). Despite similar levels of synovial macrophage infiltration and similar clinical responses to TNF blockade in both diseases, SpA is characterised by a more pronounced infiltration with alternatively activated CD163+ macrophages and ongoing osteoproliferation. This study aimed to investigate whether these differences were related to a differential expression and/or function of TNF between both diseases.

Methods Expression of transmembrane TNF (tmTNF) and soluble TNF (sTNF) was measured in IFN- $\gamma$, IL-4 or IL-10 polarised macrophages obtained from healthy donors. Expression of TNF and its receptors was measured in synovial fluid (SF) and synovial tissue biopsies (ST) of actively inflamed knee joints of SpA and RA patients. Mice transgenically overexpressing tmTNF (TgA86) were evaluated for spondylitis and arthritis. 
Results In vitro polarisation with IL-10 specifically induced the expression of CD163 on macrophages, mimicking the phenotype in SpA synovitis. IL-10 and IL-4 polarised macrophages secreted less TNF than classically, IFN- $\boldsymbol{\gamma}$-polarised macrophages $(p<0.05)$. In contrast, IL-4 polarised macrophages expressed more tmTNF compared to the IFN- $\gamma$ polarised macrophages $(p<0.05)$, with a similar trend for IL-10 polarised macrophages. In line with these in vitro data, the sTNF SF levels were significantly lower in SpA compared to RA $(p=0.01)$ despite similar TNF mRNA levels in ST. This was not related to higher expression of TNF receptors as both TNFR1 and TNFR2 were similarly expressed in ST, both at protein and mRNA levels. On the contrary, the SF levels of sTNFR1 and sTNFR2, which are both cleaved from the cell membrane by the same enzyme as tmTNF, were even decreased in SpA versus RA. Investigating the potential pathophysiological role of relative overexpression of tmTNF versus sTNF in vivo, clinical analysis revealed that tmTNF transgenic mice developed arthritis, resulting in deformation and loss of grip strength, and spondylitis as evidenced by crinkled tails with a $100 \%$ incidence. Axial and peripheral joint inflammation was confirmed by histology. In contrast to mouse strains overexpressing sTNF, tmTNF tg mice did not develop systemic disease and weight loss and showed clear signs of osteoproliferation on histology.

Conclusions tmTNF is relatively overexpressed by CD163+ alternatively polarised macrophages in SpA synovitis and leads to an axial and peripheral SpA phenotype in transgenic mice.

\section{A2.16 SYNOVIAL FLUID NEUTROPHILS UNDERGOING NETOSIS CONTRIBUTE TO JOINT INFLAMMATION BY PRODUCING CITRULLINATED AUTOANTIGENS}

doi:10.1136/annrheumdis-2013-203215.16

${ }^{1}$ Julia Spengler, 'Bozo Lugonja, ${ }^{2}$ Andrew Creese, ${ }^{3}$ Anthony Nicholas, ${ }^{2}$ Mike Milward, 'Mark Pearson, ${ }^{1}$ Christopher D Buckley, ${ }^{1}$ Andrew Filer, ${ }^{1}$ Karim Raza, ${ }^{2}$ Paul R Cooper, ${ }^{2}$ lain LC Chapple, 'Dagmar Scheel-Toellner. 'Centre for Translational Inflammation Research, School of Immunity \& Infection, College of Medical \& Dental Sciences, University of Birmingham Research Laboratories, Queen Elizabeth Hospital, Birmingham B15 2WD; 'University of Birmingham, School of Dentistry, St. Chads Queensway, Birmingham B4 6NN, UK; '3niversity of Alabama at Birmingham, Birmingham AL 35294, USA.

Objectives Anti-citrullinated protein antibodies (ACPA) are characteristically detected in patients with rheumatoid arthritis (RA) and growing evidence suggests that they are involved in disease pathogenesis. During disease flares large numbers of neutrophils enter the joint space of RA patients. These cells can extrude genomic DNA in an active process termed NETosis. NETosis critically depends on histone citrullination by peptidyl deiminase 4 (PAD4). Here we tested the hypothesis that activation and release of PAD4 during NETosis contributes to the production of autoantigens in the inflamed joint.

Materials and Methods Levels of extracellular DNA in the synovial fluid $(\mathrm{SF})$ of patients with RA $(\mathrm{n}=23)$ and osteoarthritis $(\mathrm{OA})$ $(n=15)$ or crystal arthritis $(n=6)$ were quantified using the non cell-permeable DNA dye SYTOX Green. PAD4, neutrophil elastase, citrullinated proteins and bound human IgG were labelled in association with DNA on smear preparations of RA SF and detected by immunofluorescence. NETs from SF and in vitro stimulated neutrophils were isolated and western blotting and mass spectrometry were used to identify proteins released during NET formation and proteins co-fractionated with NETs. PAD enzymatic activity was determined after NETosis in vitro and in the SF of patients with RA $(\mathrm{n}=9)$ and $O A(\mathrm{n}=9)$.

Results Extracellular DNA was detected in SF from patients with RA and crystal arthritis at significantly higher levels than in OA SF (RA versus OA $p<0.0001$ ). Association of neutrophil elastase with decondensed DNA both on smear preparations and in NETs isolated from RA SF suggests that the extracellular DNA is derived from
NETosis. On smear preparations PAD4 co-localised with decondensed chromatin in the SF of RA patients. PAD2 and PAD4 are released into the supernatant of in vitro stimulated neutrophils and were detected in NET fractions isolated from RA SFs. PAD enzymatic activity was released from neutrophils during in vitro activated NETosis while PAD activity in SF from RA patients was significantly higher than in OA patients $(p<0.001)$. The products of PAD activity, citrullinated proteins, were released into the supernatant and co-fractionated with extracellular DNA during in vitro NETosis. Furthermore, citrullinated proteins and bound human immune complexes were detected on NETs isolated from RA SF.

Conclusions These findings suggest that neutrophil NETosis, as a source of extracellular activated PAD enzymes, is involved in the production of auto-antigens in the joints of RA patients. In ACPA positive patients the resulting immune complexes can contribute to the perpetuation of the inflammatory response.

\section{A2.17 SYNOVIOCYTES CHANGE PHENOTYPE AND FUNCTION AFTER TREG-DEPLETION IN ARTHRITIC MICE}

doi:10.1136/annrheumdis-2013-203215.17

${ }^{1} \mathrm{M}$ Böttcher, ${ }^{1} \mathrm{I}$ Irmler, ${ }^{2} \mathrm{C}$ Wunrau, ${ }^{2} \mathrm{~A}$ Korb-Pap, ${ }^{3} \mathrm{G}$ Schett, ${ }^{2} \mathrm{~T}$ Pap, ${ }^{1} \mathrm{~T}$ Kamradt. ${ }^{1}$ University Hospital Jena, Institute of Immunology, Jena, Germany; ${ }^{2}$ University Hospital Muenster, Institute of Experimental Musculoskeletal Medicine, Muenster, Germany; ${ }^{3}$ University Hospital Erlangen, Department of Internal Medicine-3 and Institute for Clinical Immunology, Erlangen, Germany

Purpose/Objective Immunizstion with Glucose-6-phosphate isomerase (G6PI) induces arthritis in susceptible strains of mice. Depletion of regulatory $\mathrm{T}$ cells (Tregs) prior to immunisation switches the usually acute, self-limiting course to a non-remitting, destructive arthritis. This provides a possibility to study molecular switches for the transition from acute, self-limiting to chronic, destructive arthritis within one mouse model.

To examine the role of fibroblast-like synoviocytes (FLS), which are known to modulate immune responses via the production of pro- and anti-inflammatory mediators, the phenotype and function of FLS from mice with either acute, self-limiting or non-remitting, destructive arthritis was studied.

Materials and Methods FLS from DBA/ 1 mice that developed either the acute or the chronic form of arthritis were isolated from joints over a time course of 56 days. To investigate the phenotype of FLS ELISA studies as well as zymography have been performed. For the functional clarification of those cells the matrix-associated transepithelial resistance invasion (MATRIN) assay and a cartilage attachment assay have been used. Furthermore, FLS have been transferred in vivo into the knee joints of immunodeficient mice and the joints have been scored histologically.

Results FLS from Treg-depleted mice produced significantly more cytokines (e.g. Interleukin 6 (IL-6)) upon stimulation with other cytokines, growth factors and TLR ligands. This increased susceptibility to cytokine stimulation in chronic animals compared to acute ones is observable throughout the disease course (56 days). Furthermore, the secretion and activity of matrix metalloproteases (MMPs) was enhanced in the FLS from chronic mice compared to samples from acute ones. Additional functional differences include the collagen-destructive potential and the potential to attach and eventually invade wild type cartilage. Here, FLS from Treg-depleted chronic arthritic mice showed a higher invasive and destructive potential Ultimately, FLS from Treg-depleted mice were able to destroy cartilage in immunodeficient mice.

Conclusions Our results are compatible with the hypothesis that uninhibited inflammation in the early phase of Treg-depleted mice causes the acquisition of an autonomously aggressive phenotype of synoviocytes which contribute to the switch from acute to chronic arthritis even in the absence of late support from $\mathrm{T}$ and B lymphocytes. 